Nota de la editora (español)

Nota de l'Editora (Català)

Editor's Note (English)

Lettre de l'éditrice (Français)

\title{
Nota de la Editora (Español)
}

Nuestro último volumen de este año 2013 ofrece una interesante variedad de temas y enfoques para la enseñanza y el aprendizaje de la lengua y la literatura. Nuestro autor invitado, el doctor Selim Ben Said, del Instituto Nacional de Educación de Singapur explora la mediación de la identidad en el proceso de formación de una docente de lenguas. En concreto, el autor examina la manera en que una futura docente despliega "discursos de socialización", en un reflexión compartida con su nueva "comunidad de práctica" (Wenger, 1998), con el fin de dar legitimidad a su posición como miembro de dicha comunidad. Mediante extractos de entrevistas semi-estructuradas, que permiten analizar las narrativas de la estudiante futura docente sobre de su propia formación, Ben Said muestra las tensiones entre las idealizaciones de la futura docente respecto de la enseñanza y su experiencia real, mostrando así la existencia de un espacio mediador entre la estudiante y lo Wasko y Faraj (2000) llaman "conocimiento incardinado en una comunidad", en este caso, la comunidad de docentes de lenguas.

En el siguiente artículo, Özkan Gürses y Adigüzel abordan los efectos de la enseñanza de enseñanza de estrategias de lectura en los avances de estudiantes de un curso preparatorio de francés como lengua extranjera. Mediante un enfoque de investigación-acción, los autores recolectaron datos a través de la técnica "pensar en voz alta" antes y después de la implementación de estrategias de lectura específicas. El siguiente artículo, redactado por Anguera Cerarols, se expone un proyecto de investigación sobre la enseñanza del inglés como lengua extrajera en educación primaria, cuyo objetivo es la adquisición de competencias múltiples a partir de un enfoque por proyectos. El autor examina los logros de los alumnos desde una perspectiva multidisciplinar, centrándose, en particular, sobre los aprendizajes de contenidos de ciencias sociales, a través del uso de lenguas extranjeras potenciado por el recurso a tecnologías on-line entre participantes. En una dirección similar (aunque con atención en el docente en este caso), Fuentes presenta un estudio de caso sobre actitudes de docentes, contrastadas con resultados de aprendizaje previos en una clase de ciencias en que se emplea el inglés como medio de instrucción. 
Terminamos nuestro último volumen del año con la reseña de María Elena Molina sobre el libro Escribir para aprender. Disciplinas y escritura en la escuela secundaria, de Federico Navarro y Andrea Revel Chion (Buenos Aires: Paidós). Por último, Mireia Sala Bertran entrevista a María del Carmen Lorenzatti acerca de sus puntos de vista sobre los nuevos estudios de alfabetización y la teoría de la actividad, tal como se aplica en las prácticas sociales escolares.

Mis mejores deseos para el próximo año.

Dra. Melinda Dooly Owenby

17 diciembre de 2013

\section{Referencias bibliográficas}

Wasko, M.; y Faraj, S. (2000). 'It is what one does': why people participate and help others in electronic communities of practice. Journal of Strategic Information Systems, 9 (2-3), 155-173.

Wenger, E. (1998). Communities of practice: Learning, meaning, and identity. Cambridge: Cambridge University Press.

\section{Créditos}

Las ilustraciones para las portadas de cada edición son diseñadas por estudiantes de EINA (Escuela de Diseño y Arte, Barcelona), dentro del programa del posgrado en Ilustración Creativa, dirigido por Sonia Pulido, profesora de Ilustración de Prensa.

\section{Los revisores del volumen 6.4}

La editora agradece a las siguientes personas su contribución a la preparación del Volumen 6.4:

Mercè Bernaus, Victor Corona, Christiane Dalton-Puffer, Melinda Dooly, Oriol Guasch, Gosia Kurek, François Mangenot, Marie Nadeau, Sonia Sierra y Steve Walsh. 


\section{Nota de l'editora (català)}

El nostre darrer volum d'aquest any 2013 ofereix una interessant varietat de temes i enfocaments per a l'ensenyament i l'aprenentatge de la llengua i la literatura. El nostre autor convidat, el doctor Selim Ben Said, de l'Institut Nacional d'Educació de Singapur explora la mediació de la identitat en el procés de formació d'una docent de llengües. En concret, l'autor examina la manera en què una futura docent desplega "discursos de socialització", en un reflexió compartida amb la seva nova "comunitat de pràctica" (Wenger, 1998), per tal de donar legitimitat a la seva posició com a membre de aquesta comunitat. Mitjançant extractes d'entrevistes semi-estructurades, que permeten analitzar les narratives de la estudiant futura docent sobre de la seva pròpia formació, Ben Said mostra les tensions entre les idealitzacions de la futura docent respecte de l'ensenyament i la seva experiència real, mostrant així l'existència de un espai mediador entre la estudiant i el Wasko i Faraj ( 2000 ) anomenen "coneixement incardinat en una comunitat", en aquest cas, la comunitat de docents de llengües.

En el següent article, Özkan Gurses i Adigüzel aborden els efectes de l'ensenyament d'ensenyament d'estratègies de lectura en els avenços d'estudiants d'un curs preparatori de francès com a llengua estrangera. Mitjançant un enfocament d'investigació- acció, els autors van recol-lectar dades a través de la tècnica "pensar en veu alta " abans i després de la implementació d'estratègies de lectura específiques. En el següent article, redactat per Anguera Cerarols, s'exposa un projecte d'investigació sobre l'ensenyament de l'anglès com a llengua estrangera en educació primària, l'objectiu del qual és l'adquisició de competències múltiples a partir d'un enfocament per projectes. L'autor examina els èxits dels alumnes des d'una perspectiva multidisciplinària, centrant-se, en particular, en els aprenentatges de continguts de ciències socials, a través de l'ús de llengües estrangeres potenciat pel recurs a tecnologies on-line entre participants. En una direcció similar (encara que amb atenció al docent en aquest cas), Fuentes presenta un estudi de cas sobre actituds de docents, contrastades amb resultats d'aprenentatge previs en una classe de ciències en què s'empra l'anglès com a mitjà d'instrucció .

Acabem el nostre últim volum de l'any amb la ressenya de María Elena Molina sobre el llibre Escribir para aprender. Disciplinas y escritura en la escuela secundaria, de Federico Navarro y Andrea Revel Chion (Buenos Aires: Paidós). Finalment, Mireia Sala Bertran entrevista Maria del Carme Lorenzatti sobre els seus punts de vista 
respecte dels nous estudis d'alfabetització i la teoria de l'activitat, tal com s'aplica en les pràctiques socials escolars.

Els meus millors desitjos per al proper any.

Dra. Melinda Dooly Owenby

17 de desembre de 2013

\section{Referències Bibliogràfiques}

Wasko, M.; i Faraj, S. (2000). 'It is what one does': why people participate and help others in electronic communities of practice. Journal of Strategic Information Systems, 9 (2-3), 155-173.

Wenger, E. (1998). Communities of practice: Learning, meaning, and identity. Cambridge: Cambridge University Press.

\section{Crèdits}

Les il·lustracions per a les portades de cada edició són dissenyades per estudiants d'EINA (Escola de Disseny i Art, Barcelona), dins el programa del postgrau d'Il·lustració Creativa, dirigit per Sonia Pulido, professora d'Il-lustració de Premsa.

\section{Els revisors de volum 6.4:}

Mercè Bernaus, Victor Corona, Christiane Dalton-Puffer, Melinda Dooly, Oriol Guasch, Gosia Kurek, François Mangenot, Marie Nadeau, Sonia Sierra i Steve Walsh. 


\section{Editor's Note (English)}

Our last volume of this year 2013 offers an interesting array of topics and approaches to language and literature teaching and learning. Our guest author, Dr. Selim Ben Said of the National Institute of Education of Singapore explores the mediation of identity during the process of becoming a language teacher. Specifically, the author examines the way in which a student-teacher deploys "discourses of socialization" (shared repertoire) of her new "Community of Practice" (Wenger, 1998) in order to make her position as a member of that community legitimate. Using semi-structured interview excerpts to analyse the student-teacher's narrative of her own development, Ben Said probes the tensions between her idealized concepts of teaching and her actual experience, thus providing a mediating space for the student-teacher to activate what Wasko and Faraj (2000) call "knowledge embedded in a community" -in this case, the community of language teachers.

Our next article, by Özkan Gürses and Adigüzel addresses the effects of reading strategy instruction on students' achievement levels in a foreign language. Taking an action research approach to study a preparatory class of French as a foreign language, the authors collected data through a "think-aloud technique" before and after specific reading strategy interventions. The following article, by Anguera Cerarols, reports on a research project of English as a Foreign Language primary education that aimed to integrate multiple competence acquisition in a project-based language learning intermediation. The author examines the students' achievement from a multidisciplinary perspective, focusing, in particular on student gains of content learning in Social Sciences, facilitated through technology-enhanced foreign language use with online partners. Along similar lines (although with a focus on the teacher rather than students), Fuentes presents a case study of teachers' attitudes -cross-referenced to previously evaluated student output- during a Science through English classroom.

We end our last volume of the year with a review written by Maria Elena Molina of the book Escribir para aprender. Disciplinas y escritura en la escuela secundaria, by Federico Navarro y Andrea Revel Chion (Buenos Aires: Paidó). And lastly, Mireia Sala Bertran interviews María del Carmen Lorenzatti about her views on the New Literacy Studies and Activity Theory, as applied to social practices in the school. 
Best wishes for the upcoming year.

Dr. Melinda Dooly Owenby

17 December 2013

\section{Works Cited:}

Wasko, M.; \& Faraj, S. (2000). 'It is what one does': why people participate and help others in electronic communities of practice. Journal of Strategic Information Systems, 9 (2-3), 155-173.

Wenger, E. (1998). Communities of practice: Learning, meaning, and identity. Credits: Cambridge: Cambridge University Press.

Illustrations for the covers of each issue are designed by students of EINA (Escola de Disseny i Art, Barcelona) studying in the postgraduate course 'Il-lustració Creativa', under the direction of Sonia Pulido, teacher of Illustration for Publishing Media.

\section{Reviewers for Volume 6.4}

The editor would like to thank the following reviewers for their contribution to the preparation of Volume 6.4:

Mercè Bernaus, Victor Corona, Christiane Dalton-Puffer, Melinda Dooly, Oriol Guasch, Gosia Kurek, François Mangenot, Marie Nadeau, Sonia Sierra and Steve Walsh. 


\section{Lettre de l'éditrice (Français)}

Notre dernier volume de l'année 2013 offre une intéressante variété de sujets et d'approches sur l'enseignement et l'apprentissage de la langue et de la littérature. Notre auteur invité, le professeur Selim Ben Said, de l'Institut National de l'Éducation de Singapour explore la médiation de l'identité dans le processus de formation d'une enseignante de langues. Plus précisément, l'auteur examine la manière dont une future enseignante de langues partage un « discours de socialisation » dans sa nouvelle «communauté de pratique » ( Wenger, 1998 ), afin de donner une légitimité à sa position en tant que membre de cette communauté. En ayant recours à des extraits d'interviews semi- structurées, qui analysent les récits de l'enseignante en formation à propos de son parcours, Ben Said montre les tensions entre l'idéalisation de l'éducation de cette étudiante et son expérience réelle, montrant ainsi l'existence d'un espace de médiation entre l'enseignante en formation et ce que Wasko et Faraj ( 2000 ) appellent « les connaissances intégrées dans une communauté », la communauté des enseignants de langues, dans ce cas.

Dans l'article suivant, Özkan Adiguzel Gürses abordent les effets des stratégies d'enseignement de la lecture chez des élèves d'un cours préparatoire de français comme langue étrangère. En utilisant une approche de recherche-action, les auteurs ont recueilli des données à travers la technique «penser à haute voix » avant et après la mise en œuvre de stratégies de lecture spécifiques. L'article suivant, écrit par Anguera Cerarols, présente un projet de recherche sur l'enseignement de l'anglais comme langue étrangère dans l'enseignement primaire, dont l'objectif est l'acquisition de compétences multiples à partir d'une approche par projet. L'auteur examine les résultats des élèves dans une perspective pluridisciplinaire, en se concentrant en particulier sur l'apprentissage des sciences sociales, à travers de l'utilisation d'une langue étrangère favorisé par l'utilisation de technologies qui permettent la communication en ligne entre les participants. Dans le même sens (dans ce cas, cependant, avec un focus sur les enseignants), Fuentes présente une étude de cas sur les attitudes des enseignants, en contraste avec les résultats d'apprentissage précédents dans une classe de sciences enseignées en anglais .

Nous terminons le dernier volume de l'année par un compte rendu rédigé par Maria Elena Molina sur le livre Escribir para aprender. Disciplinas y escritura en la escuela secundaria, de Federico Navarro et Andrea Revel Chion (Buenos Aires: 
Paidós). Finalement, Mireia Sala Bertrán mène un entretien avec María del Carmen Lorenzatti sur son point de vue à propos des nouvelles études sur l'alphabétisation et la théorie de l'activité, telle qu'elle est appliquée dans les pratiques sociales de l'école. Avec mes meilleurs désirs pour la nouvelle Année.

Melinda Dooly

Décembre 2013

\section{Références:}

Wasko, M.; et Faraj, S. (2000). 'It is what one does': why people participate and help others in electronic communities of practice. Journal of Strategic Information Systems, 9 (2-3), 155-173.

Wenger, E. (1998). Communities of practice: Learning, meaning, and identity. Cambridge: Cambridge University Press.

\section{Crédits}

Les illustrations des couvertures de chaque volume ont été conçues par des étudiants de l'école EINA (Escola de Disseny i Art, Barcelona), dans le cadre du programme d'études supérieures 'Illustration créative', sous la direction de Sonia Pulido, professeure d'illustration de presse.

\section{Au comité de lecture du volume 6.4}

L'éditrice remercie les personnes suivantes pour leur contribution à la préparation $\mathrm{du}$ Volume 6.4:

Mercè Bernaus, Victor Corona, Christiane Dalton-Puffer, Melinda Dooly, Oriol Guasch, Gosia Kurek, François Mangenot, Marie Nadeau, Sonia Sierra et Steve Walsh. 